\title{
On Semi-Classical Approach to Materials Electronic Structure
}

\author{
Levan Chkhartishvili, ${ }^{1, *}$
}

\author{
${ }^{1}$ Department of Engineering Physics, Georgian Technical University, Tbilisi 0160, Georgia \\ ${ }^{2}$ Boron and Powder Composite Materials Laboratory, Ferdinand Tavadze Metallurgy and Materials Science \\ Institute, Tbilisi 0186, Georgia
}

\begin{abstract}
Materials atomic structure, ground-state and physical properties as well as their chemical reactivity mainly are determined by electronic structure. When first-principles methods of studying the electronic structure acquire good predictive power, the best approach would be to design new functional materials theoretically and then check experimentally only most perspective ones. In the paper, the semi-classical model of multi-electron atom is constructed, which makes it possible to calculate analytically (in special functions) the electronic structure of atomic particles themselves and materials as their associated systems. Expected relative accuracy makes a few percent, what is quite acceptable for materials science purposes.
\end{abstract}

Keywords: Atom, Material, Electronic structure, First-principles method, Semi-classical approximation.

\section{INTRODUCTION}

Electronic structure, i.e. electron energy spectrum plus electron density distribution in space, plays a fundamental role in determining the materials cohesion and equilibrium atomic structure, as well as their phase transitions, physical properties, and chemical reactivity [1].

The microscopic view of materials as composed of interacting atoms, which in turn are the bounded systems of nuclei and electrons, aiming to explain all the properties of materials purely theoretically - based only on quantum mechanics without any adjustable semiempirical parameters - is called as first-principles approach. Using the first-principles methods acquired good predictive power to design new functional materials can be preferable compared to their experimental search. Point is that there is suggested a huge number of materials potentially useful in various technologies and industries and then their laboratory preparation and checking whether possess the desired properties are too expensive and time consuming. Therefore, a better approach would be to design new materials theoretically, in a "virtual laboratory", and then investigate experimentally only most perspective ones.

As is known, in materials only a part of electrons called valence electrons are actively involved in the formation of interatomic chemical bonds, whereas the rest of electrons called core electrons together with

*Address correspondence to this author at the Department of Engineering Physics, Georgian Technical University, 77 Merab Kostava Avenue, Tbilisi 0160, Georgia;

E-mails: levanchkhartishvili@gtu.ge and chkharti2003@yahoo.com nuclei remain in states almost indistinguishable from their states in corresponding isolated atoms. Therefore, constituent atoms largely retain their individuality within the material and its treatment as a structure of atomlike systems seems to be quite acceptable.

This fact explains the successful practice of theoretical description of many materials by the LCAO (Linear-Combination-of-Atomic-Orbitals) method - see e.g. [2] (for the origins see [3, 4] as well), which is looking for the electronic subsystem wave function as a linear combination of atomic-like orbitals. Both the accuracy and range of applicability of the LCAO method are greatly dependent of effective expression of the set of constituent atoms electronic orbitals used in trail. Since the standard first-principle quantumchemical methods give AOs in numerical form, the matrix elements of the corresponding secular equation determining the material electronic structure in the LCAO approach usually have to be found by numerical integration as well.

In the present work, there is developed the semiclassical model of multi-electron atom leading to the analytical expressions of AOs through special functions. Using them, again in special functions one can calculate the electronic structure of atomic particles themselves and their associations in form of materials.

\section{RELATIVISTIC GENERALIZATION OF BOHR SEMI-CLASSICAL MODEL}

Since electrons in low-lying orbits in heavy atoms move with relativistic velocities, the non-relativistic analysis is insufficient to estimate the minimum radius of the electron orbits: it is necessary to involve relativistic mechanics. For this reason, below we give 
relativistic generalization of the semi-classical nonrelativistic Bohr model for hydrogen-like atoms.

Considering the nucleus as a point particle of infinite mass and, consequently, being in the rest and having the positive charge of $+\mathrm{eZ}$ ( $\mathrm{e}$ is the elementary electric charge, and $Z$ is the nucleus charge number), we estimate the allowed semi-classical values of the radius $r$ of stationary circular orbit of relativistic electron (with the negative charge of -e) bounded by electrical interaction with nucleus located in the center of the orbit. Let $v$ be the magnitude of the velocity of the electron in its orbit. The assumption of stationary motion means its constancy: $v=$ const. Since the centripetal acceleration of such electron is caused by the nucleus Coulomb attractive force, the relativistic equation of motion is obtained in the following form (in Gauss units):

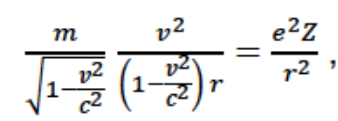

where $\mathrm{c}$ denotes the light speed and $\mathrm{m}$ is the electron rest mass.

In the spirit of the "old quantum mechanics", we require that in the relativistic hydrogen-like atom the allowed electron orbit length should be a multiple integer of the de Broglie wavelength of the moving in this orbit electron:

$\frac{2 \pi r}{n}=\frac{2 \pi \hbar}{\frac{m v}{\sqrt{1-\frac{v^{2}}{c^{2}}}}}$.

Here $\hbar$ denotes the Planck constant and is introduced the quantum number $n$ taking natural values:

$n=1,2,3, \ldots$.

Two equations, (1) and (2), make up the system of equations for two unknowns $r$ and $v$. Excluding $r$ from them, we obtain quadratic equation defining $v$ :

$\left(\frac{v}{c}\right)^{2}+\frac{n}{\alpha Z}\left(\frac{v}{c}\right)-1=0$. is:

Having a physical meaning, i.e. positive, solution $v_{\mathrm{n}}$

$$
\frac{v_{n}}{c}=\sqrt{1+\frac{1}{\beta_{n}}}-\sqrt{\frac{1}{\beta_{n}}} .
$$

Here we introduced the electron orbit parameter $\beta_{n}=\frac{4 \alpha^{2} Z^{2}}{n^{2}}$

determined by so-called fine-structure constant:

$\alpha=\frac{e^{2}}{\hbar c} \approx \frac{1}{137.04}$.

The value of $\beta_{\mathrm{n}}$ can serve as a criterion for the relativism of the electron motion in its orbit. As expected, in the non-relativistic limit, which corresponds to the condition $\beta_{\mathrm{n}} \ll 1$, the solution (5) gives velocity of the electron in Bohr model orbit:

$\frac{v}{v_{\text {Bohr }}} \approx \frac{Z}{n}$,

where $v_{\text {Bohr }}=\frac{e^{2}}{\hbar} \approx 2.2 \cdot 10^{6} \frac{\mathrm{m}}{\mathrm{s}}$ is the electron velocity in the hydrogen atom first Bohr orbit. This indeed is a non-relativistic velocity, $\frac{v_{\mathrm{Bohr}}}{c}=\alpha \approx \frac{1}{137.04} \ll 1$ and then explains success of the non-relativistic Bohr model.

Now from equations (2) and (5) it is easy to find the radii $r_{\mathrm{n}}$ of allowed electron orbits:

$\frac{r_{n}}{r_{\mathrm{Bohr}}}=\frac{n^{2}}{Z} \sqrt{\frac{\sqrt{1+\beta_{n}}+1}{2}}$.

Here $r_{\text {Bohr }}=\frac{\hbar^{2}}{e^{2} m} \approx 5.3 \cdot 10^{-11} \mathrm{~m}$ denotes so-called Bohr radius, the hydrogen atom first Bohr orbit radius. From formula (9), in the non-relativistic limit $\beta_{n} \ll 1$ we have $\frac{r_{n}}{r_{\text {Bohr }}} \approx \frac{n^{2}}{Z}$ as one would expect.

And finally, we set the semi-classical electron energy levels $E_{n}$ in relativistic hydrogen-like atom:

$E_{n}=\frac{m c^{2}}{\sqrt{1-\frac{v_{n}^{2}}{c^{2}}}}-\frac{e^{2} Z}{r_{n}}$

or

$\frac{E_{n}}{m c^{2}}=\sqrt{\frac{\sqrt{1+\beta_{n}}+1}{2}}-\frac{1}{2} \sqrt{\frac{\beta_{n}\left(\sqrt{1+\beta_{n}}-1\right)}{2}}$.

In the non-relativistic limit, $\beta_{n} \ll 1$, it is permissible to decompose the right-hand side of this relation into series in powers of $\beta_{n}$ and retain only members of zero and first orders:

$E_{n} \approx m c^{2}-\frac{e^{4} m Z^{2}}{2 \hbar^{2} n^{2}}$. 
These two terms exactly coincide, respectively, with the rest energy of the electron and the non-relativistic energy level of the electron-state with the number of $n$ in the hydrogen-like atom. However, the following terms of the decomposition turn out to be inaccurate: they do not coincide precisely with the quasi-relativistic corrections to the non-relativistic eigenvalues according to the Dirac relativistic wave equation for hydrogen-like atom. This is explained by the impossibility of taking into account in the semi-classical approximation the electron's intrinsic mechanical momentum - spin.

At given $\mathrm{Z}$, the minimum radius corresponds to the first orbit with $n=1$. On the other hand, the radius of the orbit decreases with increasing charge number $Z$. If this number is so large that $\alpha Z>1$, i.e. if $Z>137$, then the solution of the Dirac wave equation with the Coulomb potential does not satisfy the physical boundary condition at the center of the field: $r \rightarrow 0$ [5]. Consequently, the values $Z_{\max }=137$ and, accordingly, $\beta_{n \text { max }}=4$ are extremely possible values from the point of view of the atom stability. Although, in real atoms, the nuclei themselves become unstable at much lower $Z$ and $\beta_{n}$. Finally, we get the desired estimate of the minimum radius of the relativistic electron orbit in atom: $r_{\min } \approx \frac{r_{\text {Bohr }}}{137.04} \sqrt{\frac{\sqrt{5}+1}{2}} \approx 4.9 \cdot 10^{-13} \mathrm{~m}$.

Our relativistic model of single-electron atom seems to be the most direct generalization of the well-known non-relativistic Bohr model of hydrogen atom [6-8].

\section{KEY ASSUMPTIONS OF SEMI-CLASSICAL MODEL OF MULTI-ELECTRON ATOM}

\subsection{Stationary State}

Atomic nuclei are the bounded systems of nucleons - protons and neutrons. The presence of at least one proton is obligatory for a nucleus to have a non-zero electric charge. As the nucleon mass in $\sim 2000$ times exceeds the electron mass, the mass of an atomic electron is almost negligible compared to the mass of atomic core, i.e. the system of nucleus and the rest of electrons. It is clear that such approximation "works" better for heavier atoms. Assuming the nucleus mass to be infinite, atom can be imagined as a system of a positively charged nucleus fixed in the rest and negatively charged electrons moving around in closed orbits. Each atomic electron is affected by approximately stationary SCF (Self-Consistent-Field) consisted from stationary electric field of the nucleus and time-averaged superposition of electric fields of atomic core's moving electrons.
As single-electron wave equation with stationary binding potential leads to discrete energy eigenvalues, by the introducing atomic SCF conception multielectron atom problem is reduced to the determination of its electronic structure - single-electron AOs and corresponding electron energy levels.

Initially, a relativistic correction to a non-relativistic atomic electron level appears only in the second order with respect to the $\frac{v}{c}$ ratio. According to the perturbation theory, the relativistic correction of order of $\frac{v^{2}}{c^{2}}$ leads to so-called fine-structure of the electronic energy spectrum (see e.g. [9]). In parallel, this correction accounts the first-order electron-spin effects. As for the correction of order of $\frac{v^{3}}{c^{3}}$, it takes into account the electron radiation, what is incompatible with its stationary motion. Thus, it makes no sense to take into account relativistic corrections of orders higher than 2. Note, the second-order correction to the energy corresponds to the first-order correction to the wave function. And since there are no first-order relativistic corrections, any relativistic corrections to the wave function should be neglected as well.

\subsection{Spherical Symmetry}

According to modern concepts, electrons are truly point-like particles. Can one consider the nuclei as material points too? If so, the nucleus fixed in a point should act as the spherical symmetry center of the intra-atomic SCF. To find the answer to the question posed, below we evaluate the atomic nucleus maximum radius to compare it with minimum radius of the relativistic electron orbit.

Due to the almost constant density of nuclear matter, the radius of the nucleus was found [10] to be approximately proportional to the cubic root of the atomic number $A$, the sum of numbers of protons $\mathrm{Z}$ and neutrons in the nucleus: $R \approx R_{0} A^{1 / 3}$ with the averaged value of the proportionality coefficient $R_{0} \approx 1.2 \cdot 10^{-15} \mathrm{~m}$. Note that here $\mathrm{R}$ is the root-meansquare charge radius of the nucleus, not a geometric characteristic. For the most stable nuclei, the ratio of numbers of neutrons and protons is estimated as $1+0.015 A^{2 / 3}$. In this way, we get the equation: $A \approx Z\left(2+0.015 A^{2 / 3}\right)$. The integer part of its solution at $Z_{\text {max }}=137$ gives estimation for the maximum atomic number: $A_{\max }=382$. Then $R_{\max } \approx 8.7 \cdot 10^{-15} \mathrm{~m}$. It turns out that the ratio of the maximum possible nucleus radius to the minimum electron orbit radius in atoms is much less than 1: $\frac{R_{\max }}{r_{\min }} \approx 0.018 \ll 1$. Therefore, even for an extremely heavy nucleus its charge radius is significantly smaller compared with the 
characteristic distance to electron in lowest-lying orbit. For the overwhelming majority of electron-states in atoms with stable nuclei, similar conditions are fulfilled in much better.

Thus, atomic nuclei can be assumed to be point particles. The nucleus representation as a fixed point electric charge means that the intra-atomic electric SCF is of spherical symmetry with the center at nucleus. The contribution from the point nucleus Coulomb-like field certainly satisfies the spherical symmetry condition. However, spherical symmetry is not obvious for electronic contributions. The fact is that the AOs themselves, which are found by solving the wave equation with radial potential, are not spherically symmetric - in addition to the radial factor they also contain the angular factor. Therefore, to achieve selfconsistency, it is necessary that when calculating the atomic SCF the partial electron densities be averaged over directions.

\subsection{Semi-Classical Approximation}

Atomic absorption and emission spectra theoretically calculated assuming atomic electric field to be stationary and spherically symmetric are in agreement with all the available experimental data. As for the theoretical approaches to the problem of electronic structure of atoms and materials, among them most fruitful are various variants of the semiclassical approximation such as Bohr and ThomasFermi type models and DFT (Density-FunctionalTheory). Let describe them in brief.

It can be said that the semi-classical approximation to quantum mechanics appeared simultaneously with quantum mechanics itself. As early as in his seminal work [6] Bohr wrote: "... the dynamical equilibrium of the systems in the stationary states can be discussed by help of the ordinary mechanics ... . ... assumption seems to present itself; for it is known that the ordinary mechanics cannot have an absolute validity, but will only hold in calculations of certain mean values of the motion of the electrons. On the other hand, in the calculations of the dynamical equilibrium in a stationary state in which there is no relative displacement of the particles, we need not distinguish between the actual motions and their mean values". Standard quantum mechanics notwithstanding, Bohr's celebrated model of the hydrogen atom is still taught as a paradigmatic example of successful modeling. There is argued [11] that contrary to widespread belief Bohr's model is consistent and can be interpreted to support the moderate and selective version of the realistic description.

It was reported [12] on so-called dipole-shell model of the atom, which can be considered as a neoclassical development of the Bohr's semi-classical shell-model. In such a way, atom is represented as a classical object - set of nested each in other quasi-spheres or shells formed by circular / elliptical electron orbits. The conceptual basis of this model, which consists in using the Gauss theorem to reduce the multi-charge problem to the two-charge one, is interesting. But, we strongly disagree with the statement that such a purely classical description, completely neglecting any quantum effects, can give the exhaustive explanation of all the observed atomic phenomena. Although, it was really demonstrated how to extract useful information about the light atoms electronic structure from their classical study by numerical methods - see the example of twoelectron or helium-like atoms [13].

It is noteworthy that the Bohr's simple semi-classical model gives exact electron energy spectrum for hydrogen-like atoms. This success of the semiclassical approach is partially accidental. The fact is that the potential acting on single electron is purely Coulomb-like and the corresponding wave equation can be solved exactly. Not only for the attracting Coulomb [14], but also for any other exactly solvable bounding potential [15], the quantization rule in the initial quasi-classical or semi-classical approximation leads to exact values of discrete energy levels of the system. An attempt to directly extend the Bohr's simple semi-classical model to helium-like atoms [16] leads to the renormalization of the nuclear charge number: $Z \rightarrow Z-\frac{1}{4}$. As a result, for two-electron atomic systems $\mathrm{He}^{0}, \mathrm{Li}^{+}$, and $\mathrm{Be}^{2+}$ the obtained ground state energies differ from the experimental ones only in a few percent.

The review [17] also discussed the renaissance of semi-classical methods and described modified semiclassical concepts and their application to two-electron atoms offering the viewpoint complementary to numerical quantum-mechanical alone. In this way, it was shown how the simple interpretations of the approximate quantum numbers and propensity rules can be given in terms of a few key periodic orbits of the classical three-body problem. Instead of trying to solve the wave equation explicitly, the focus lies on the study of the classical three-body Coulomb problem and its influence on spectral features of the corresponding quantum system. This includes the puzzling existence of Rydberg series for electron-pair motion. In particular, 
by exploiting the classical information in detail, periodic orbit trace formulas are able to resolve quantitatively individual resonances and bound states from the ground state across the various two-body fragmentation thresholds.

The suitability of simple Bohr-type analytical models was also demonstrated for electron trajectories in slightly more complex systems: atoms, small molecules, and ions with two or three electrons [18]. It was shown that the classical description of periodic motion is not necessarily limited to those cases where Planck constant is assumed to tend to zero: $\hbar \rightarrow 0$. Besides, there was found a simple extension of the Bohr molecular model [19] that gives a clear physical picture of how electrons create chemical bonding. The description is surprisingly accurate providing good ground-state potential energy curves not only for hydrogen molecule $\mathrm{H}_{2}$, but also relatively complicated molecules such as $\mathrm{LiH}, \mathrm{Li}_{2}$, $\mathrm{BeH}$, and $\mathrm{He}_{2}$.

To simulate the many-electron systems such as materials, the semi-classical approximation has to be combined with the statistical one. Even the simplest Thomas-Fermi equation [20, 21] gives the distribution of intra-atomic electron density, which is suitable as initial approximation when interpreting the electromagnetic waves scattering by materials. Moreover, the methodology for describing the spectra system based on poor information allowed [22] to find single-electron densities even beyond electrons classical turning points based on Thomas-Fermi model and then use them for revealing shell-effects characteristic of many-electron quantum systems. When the number of electrons reaches about 10 , the relative differences between model results and firstprinciples ones decrease to $1-2 \%$.

Further development of the approach leads to the class of modified Thomas-Fermi equations (see e.g. [23]), which take into account various statistical and quantum corrections introduced by adding corresponding terms in the atom electron system total energy expression. They are represented by series of powers of the small parameter $\frac{1}{N^{1 / 3}}$, where $N$ denotes the number of electrons in the system. Thomas-Fermi model admits direct relativistic generalization as well. Emphasize that all these corrections are small enough to neglect their interferences.

The most consistent generalization of the ThomasFermi model is represented by the Magomedov radialstatistical model [24], in which radial wave functions corresponding to the semi-classical SCF are introduced for spherical bound multi-electron system such as atom. This approach is equivalent to the ThomasFermi model only in the limit of infinite number of electrons in the system: $N \rightarrow \infty$. Magomedov model has revealed the lost major correction $\sim \frac{1}{N^{1 / 3}}$ [25], the nature of which is purely statistical. It is associated with the replacement of the summation over quantum numbers by integration, when considering these numbers as continuous variables. The next correction, $\sim \frac{1}{N^{2 / 3}}$, basically is a statistical one as well - caused by taking into account the electron-electron exchange (Dirac correction). There are quantum and shell corrections (von Weizsacker correction and others) of the same parametric smallness, but with much smaller numerical coefficients. The introduction of the correction $\sim \frac{1}{N}$ (Amaldi correction) is caused by the necessity to exclude the nonphysical effect of electron self-action, i.e. again related to the use of statistical approximation. Thus, errors of the Thomas-Fermi-type approaches are predominantly of statistical origin, not semi-classical one.

DFT is the most modern formulation of statistical modeling of multi-electron bounded systems. The density of the total kinetic energy of the electron jellium as a function of electron density permits systematic decomposition into quasi-classical series [26] and the initial - semi-classical approximation of the series corresponds to so-called LDA (Local-DensityApproximation). The well-known success of applications practice of DFT and, in particular, LDA once again confirms the suitability of the semi-classical approaches to atoms and systems of bounded atoms, i.e. materials.

\section{CONSTRUCTING SEMI-CLASSICAL POTENTIALS AND ELECTRON ORBITALS}

Before calculating materials electronic structure in the semi-classical approximation, one needs to construct inner semi-classical potential acting on electrons of constituting atoms. Below, the term "atom" is understood to mean not only isolated neutral atoms or atomic ions, but also any of atom-like particles - the bounded system of a nucleus and electrons, which constitute "material" - the bounded system of atoms. Consider the atom containing nuclei with charge number of $Z$ and $N$ electrons. Let number electrons by the index $i$ :

$$
i=1,2,3, \ldots, N .
$$

Obviously, it is equivalent to the set of atomic quantum numbers. Denote the corresponding discrete electron energy levels by $E_{i}$ Since the motion of 
electrons in atom is assumed to be semi-classical, most of the time the $i$-electron should be near the surface of the sphere with certain radius $r_{\mathrm{i}}$. In other words, it is possible to assume that for $i$-electron radial coordinate is nearly constant $r \approx r_{i}$.

By the Gauss theorem, the stationary radial electric field on the surface of the sphere coincides with the Coulomb field created by point charge localized at the center of the sphere, the value of which is determined by the sum of electric charges inside this sphere. Therefore, the potential energies $U_{i}=U_{i}(r)$ of atomic electrons can be approximated in the Coulomb-shaped form:

$$
U_{i}(r) \approx-\frac{e^{2} Z_{i}}{r},
$$

where for $i$-electron the relation

$$
Z_{i}=Z-4 \pi \sum_{\substack{i \neq k=N \\ i \neq k=1}}^{r_{i}} d r r^{2} \rho_{k}(r)
$$

determines the effective charge number $Z_{i}$ of the nucleus shielded by all other electrons of the atom. Here functions $\rho_{i}=\rho_{i}(r)$ are the radial distributions of partial electron densities in atom.

Expressing electric field potentials acting on atomic electrons in the Coulomb-like form is too convenient because the Coulomb potential is exactly solvable and therefore it is possible to find analytical representations of density functions $\rho_{i}(r)$, parameters $r_{i}$ and $E_{i}$ and, thus, calculate the effective charge numbers $Z_{i}$. Using these values one finds explicit electron $\operatorname{AOs} \psi_{i}=\psi_{i}(\boldsymbol{r})$ :

$\psi_{i}(\boldsymbol{r})=Y_{i}(\vartheta, \varphi) R_{i}(r)$,

where radius $0 \leq r \leq \infty$, azimuth $0 \leq \vartheta \leq \pi$ and polar $0 \leq \varphi \leq 2 \pi$ angles are the spherical coordinates. In general, for wave functions corresponding spherically symmetric potential their angular parts $Y_{i}(\vartheta, \varphi)$ are expressed as spherical harmonics normalized by the condition:

$\int_{0}^{\pi} d \vartheta \sin \theta \int_{0}^{2 \pi} d \varphi\left|Y_{i}(\vartheta, \varphi)\right|^{2}=1$.

Consequently, wave functions radial parts $R_{i}(r)$, which are real functions expressed by generalized Laguerre polynomials, have to satisfy the normalization condition:

$$
\int_{0}^{\infty} d r r^{2} R_{i}^{2}(r)=1 .
$$

Then, the full solid angle $4 \pi$ averaged partial electron densities are:

$\rho_{i}(r)=\frac{R_{i}^{2}(r)}{4 \pi}$.

As for the effective charge numbers, they take the following form:

$Z_{i}=Z-\sum_{\substack{i \neq k=N \\ i \neq k=1}}^{r_{i}} d r r^{2} R_{k}^{2}(r)$.

In the full atomic radial potential

$\varphi(r)=\varphi_{\text {Nucleus }}(r)+\varphi_{\text {Electrons }}(r)$,

the nucleus Coulomb potential

$\varphi_{\text {Nucleus }}(r)=\frac{Z e}{r}$

is added with electron cloud potential $\varphi_{\text {Electrons }}(r)$ determined by Poisson's equation:

$\frac{1}{r^{2}} \frac{\partial}{\partial r}\left(r^{2} \frac{\partial \varphi_{\text {Electrons }}(r)}{\partial r}\right)=e \sum_{i=1}^{i=N} R_{i}^{2}(r)$.

As is known, the superposition of potentials of constituent atoms localized in corresponding sites of the structure serves for good initial approximation to inner potential $U=U(\boldsymbol{r})$ of the material. Thus, potential energy of electron with radius-vector $r$ in the material inner field would be:

$U(\boldsymbol{r})=-e \sum_{j} \varphi_{j}\left(\left|\boldsymbol{r}-\boldsymbol{r}_{j}\right|\right)$,

where index $j$ numbers the constituent atoms and $\boldsymbol{r}_{j}$ are the radius-vectors of sites of their localization in the material structure.

For an elemental material the initial approximation to its wave function $\psi(\boldsymbol{r})$ can be presented as linear combination of electron orbitals of the constituting element in its isolated state:

$$
\psi(\boldsymbol{r})=\sum_{j} C_{j} \psi_{j}\left(\left|\boldsymbol{r}-\boldsymbol{r}_{j}\right|\right) .
$$

Here $C_{j}$ are the constants to be determined by solving the secular equation.

Material electronic structure in initial approximation obtained in this way should be used for first approximation to functions $U(\boldsymbol{r})$ and $\psi(\boldsymbol{r})$ and so on until the iteration procedure yields the material electronic structure with desired accuracy of the calculations self-consistency. 


\section{CONCLUDING REMARKS}

Previously, author developed QCTS (QuasiClassical-Theory-of-Substance) [27-37], an original quasi-classical method for calculating ground state and electronic structure parameters of molecules, nanostructures, and crystals (the most detailed presentation of the theory see in the monograph [32]). When QCTS is based on the semi-classical parameterization of constituent atoms [38-40], electric charge density and electric field potential distributions in atoms are represented by radial step-like functions. Carrying out electronic structure calculations using this method requires the resolution of some special geometric and algebraic problems [41-47]. Mathematical aspects of the approach have been summarized in the monograph [46].

QCTS implemented [48-79] for a number of molecular systems, and nano- and crystalline materials, mainly elemental boron and boron nitride structural modifications. The values of materials ground state characteristics such as molar binding and vibrational energies, and chemical bonds length; as well as electronic structure (including the density of electronic states), discrete impurity levels of electron energies, and frequencies of localized vibrations, etc. were calculated. In addition, using same method some of isotopic effects in materials were interpreted [80-84].

The relative errors of QCTS in calculating materials energy and structural parameters do not exceed a few percent, what is quite acceptable for materials science problems. Emphasize that the ambiguous uncertainties absent if the calculations are carried out in such type semi-classical approximation. The point is that the step-like presentation of electric charge density and electric field potential distributions in atoms implies the absence of the probability of finding electrons outside the regions classically accessible to them and, consequently, the strict finiteness of atomic radii. But, at finite atomic radii the infinite series expressing the matrix elements of the secular equation turn into sums of a finite number of nonzero terms without artificial termination of series.

However, advantage of the QCTS to avoid computation uncertainties turns into its disadvantage: the lack of expressions of electric charge density and electric field potential distributions in atoms and materials by continuous functions.

Here this problem is resolved by introducing hydrogen-like orbitals for semi-classical electron states in atoms. Using them for basis set, the matrix elements of secular equation determining material electronic structure can by expressed analytically, in particular, in special functions. Thus, further decrease in the materials electronic structure semi-classical calculation errors is expected.

\section{ACKNOWLEDGEMENT}

This work was supported by the Shota Rustaveli National Science Foundation of Georgia (SRNSFG) Grant \# AR-18-1045: "Obtaining of boron carbidebased nanostructured heterophase ceramic materials and products with improved performance characteristics".

\section{REFERENCES}

[1] R. Prasad, Electronic Structure of Materials, Boca Raton, CRC Press - Taylor \& Francis Group 2014.

[2] F. Bassani, G. Pastori Parravicini, R.A. Ballinger, Electronic States and Optical Transitions in Solids, Elkins Park, Franklin Book Co. (1993)

[3] JC. Slater, GF. Koster, Simplified LCAO method for the periodic potential problem, Phys. Rev. 1954; 94(6): 14981524.

https://doi.org/10.1103/PhysRev.94.1498

[4] V. Halpern, An optimized LCAO method for crystals, J. Phys. C 1970; 3(9): 1900-1911. https://doi.org/10.1088/0022-3719/3/9/009

[5] S. Flugge, Practical Quantum Mechanics, Berlin-Heidelberg Springer-Verlag 1999.

[6] N. Bohr, I. On the constitution of atoms and molecules, Phil. Mag. Ser. 1913; 6 26(151): 1-25. https://doi.org/10.1080/14786441308634955

[7] N. Bohr, II. On the constitution of atoms and molecules, Phil. Mag. Ser. 1913; 6 26(153): 476-502. https://doi.org/10.1080/14786441308634993

[8] N. Bohr, III. On the constitution of atoms and molecules, Phil. Mag. Ser. 1913; 6 26(155): 857-875. https://doi.org/10.1080/14786441308635031

[9] PV. Elyutin, VD. Krivchenkov, Quantum Mechanics with Problems, Moscow, Publ. House Nauka 1976.

[10] BR. Martin, Nuclear and Particle Physics (An Introduction), Chichester, Wiley 2006. https://doi.org/10.1002/0470035471

[11] M. Ghins, Bohr's modeling of the atom: A reconstruction and assessment, Logique \& Analyse 2012; 218(4): 1-22.

[12] AA. Potapov, Renaissance of Classical Atom, Moscow, Publ. House Nauka 2011

[13] T. Yamomoto, K. Kaneko, Exploring a classical model of the helium atom, Prog. Theo. Phys. 1998; 100(6): 1089-1105. https://doi.org/10.1143/PTP.100.1089

[14] J. Hainz, H. Grabert, Centrifugal terms in the WKB approximation and semi-classical quantization of hydrogen, Phys. Rev. A 1999; 60(2): 1698-1701. https://doi.org/10.1103/PhysRevA.60.1698

[15] A. Comtet, AD. Bandrauk, DK. Kampbell, Exactness of semiclassical bound state energies for super-symmetric quantum mechanics, Phys. Lett. B 1985; 150(1/2/3): 159-162. https://doi.org/10.1016/0370-2693(85)90160-1

[16] B. Bagchi, P. Holody. An interesting application of Bohr theory, Am. J. Phys. 1988; 56(8): 746-747. https://doi.org/10.1119/1.15479 
[17] AA. Svidzinsky, MO. Scully, DR. Herschbach, Bohr's 1913 molecular model revisited, Proc. Natl. Acad. Sci. USA 2005; 102(34): 11985-11988. https://doi.org/10.1073/pnas.0505778102

[18] ANV. Popa, Accurate Bohr-type semi-classical model for atomic and molecular systems, Rep. Inst. Atom. Phys. 1991; E12: 1-90.

[19] G. Tanner, K. Richter, J.-M. Rost, The theory of two-electron atoms: Between ground state and complete fragmentation, Rev. Mod. Phys. 2000; 72(2): 497-544. https://doi.org/10.1103/RevModPhys.72.497

[20] LH. Thomas, The calculation of atomic fields, Proc. Cambridge Phil. Soc. 1927; 23(5): 542-548. https://doi.org/10.1017/S0305004100011683

[21] E. Fermi, Un metodo statistico per la determinazione di alcune properita dell'atomo, Atti. Accad. Naz. Lincei - Rend. Cl. Sci., Fis., Mat., Nat. 1927; 6(6): 602-607.

[22] M. Casas, A. Plastino, A. Puente, Alternative approach to the semi-classical description of $\mathrm{N}$-fermion system, Phys. Rev. A 1994; 49(4): 2312-2317. https://doi.org/10.1103/PhysRevA.49.2312

[23] DA. Kirzhnits, Yu.E. Lozovik, G.V. Shpatkovskaya, Statistical model of substance, Phys. - Uspekhi 1975; 117(1): 3-47.

[24] KM. Magomedov, On theory of atomic quasi-classical selfconsistent field, Rep. Acad. Sci. USSR 1985; 285(5): 11001115.

[25] KM. Magomedov, P.M. Omarova, Quasi-Classical Computing of Atomic Systems, Makhachkala, Dagestan Branch Acad. Sci. USSR 1989.

[26] M. Brack, The physics of simple metal clusters: Selfconsistent jellium model and semi-classical approaches, Rev. Mod. Phys. 1993; 65-I(3): 677-732.

https://doi.org/10.1103/RevModPhys.65.677

[27] LS. Chkhartishvili, Quasi-classical calculation of the crystalline band structure, Trans. Georgian Tech. Univ. 1996; 3(411): 45-52.

[28] LS. Chkhartishvili, Method of analysis of crystalline electron energy spectrum based on the quasi-classically calculated characteristics of atomic orbitals, Trans. Georgian Tech. Univ. 1997; 3(414): 205-213.

[29] LS. Chkhartishvili, Construction of the effective quasirelativistic potential for multi-electron atom by the modified Thomas-Fermi method, Trans. Georgian Tech. Univ. 1998; 2(418): 26-37.

[30] LS. Chkhartishvili, Quasi-classical parameterization of substance ground-state electronic structure, Trans. Georgian Tech. Univ. 1999; 2(426): 12-19.

[31] L. Chkhartishvili, Structural and electronic characteristics of substance within initial quasi-classical approximation, Sci. Works Tbilisi Bis. State Inst. 2003; 1: 195-224.

[32] L. Chkhartishvili, Quasi-Classical Theory of Substance Ground State, Tbilisi, Tech. Univ. Press 2004.

[33] L. Chkhartishvili, Asymptotical (quasi-classical) approach to the substance space and electronic structures, In: Proc. Int. Sci. Conf. FMNS 2, Blagoevgrad, South-Western Univ. 2005; 245-254.

[34] LS. Chkhartishvili, Analytical optimization of the lattice parameter using the binding energy calculated in the quasiclassical approximation, Phys. Solid State 2006; 48(5): 846853.

https://doi.org/10.1134/S1063783406050064

[35] L. Chkhartishvili, Quasi-Classical Method of Calculation of Structural Parameters and Electronic Energy Spectrum of Substance (Thesis Diss. Dr. Sci. Phys. \& Math.), Tbilisi, Intellect 2006.

[36] LS. Chkhartishvili, M.D. Darchiashvili, Binding energies and electron energy levels of impurity atoms in crystallographic voids, In: Nauka i inovwacja 15 (Ed.-in-Ch. S. Gomiak), Przemysl, Nauka i studia 2011; 14-24.
[37] L. Chkhartishvili, On theory of doping in nanosized crystallographic voids, Nano Studies 2012; 5: 73-84.

[38] L. Chkhartishvili, T. Berberashvili, Atoms constituting nanosystems: Quasi-classical parameterization for binding energy and electronic structure calculations, Nano Studies 2010; 1: 103-144.

[39] L. Chkhartishvili, T. Berberashvili, Intra-atomic electric field radial potentials in step-like presentation. J. Electro Magn. Anal. Appl. 2010; 2(4): 205-243. https://doi.org/10.4236/jemaa.2010.24029

[40] L. Chkhartishvili, T. Berberashvili, Again on effective electrical charge of atomic nuclei, Nano Studies 2013; 8: 267-272.

[41] LS. Chkhartishvili, Volume of the intersection of three spheres, Math. Notes 2001; 69(3): 421-428. https://doi.org/10.1023/A:1010295711303

[42] LS. Chkhartishvili, Secular equation in electron theory of substance and its complete iterative solution, Trans. Georgian Tech. Univ. 2004; 2(452): 15-22.

[43] LS. Chkhartishvili, Iterative solution of the secular equation, Math. Notes 2005; 77(1): 273-279.

https://doi.org/10.1007/s11006-005-0026-y

[44] L. Chkhartishvili, Expression of the algebraic equation roots as a power series of its coefficients, Ilia Chavchavadze Tbilis Univ. Sci. Trans. 2007; 3: 209-216.

[45] L. Chkhartishvili, Iterative determination of the algebraic equation root with highest module, Ilia Chavchavadze Tbilis Univ. Sci. Trans. 2008; 4: 72-81.

[46] L. Chkhartishvili, Iterative and Transcendental Solutions of Algebraic Equations (Monograph), Saarbrucken, Palmarium Acad. Publ. 2012.

[47] LS. Chkhartishvili, Solution of an algebraic equation using an irrational iteration function, Math. Notes 2012; 92(5): 714719.

https://doi.org/10.1134/S0001434612110132

[48] LS. Chkhartishvili, Selection of equilibrium configurations for crystalline and molecular structures based on quasi-classical inter-atomic potential, Trans. Georgian Tech. Univ. 1999; 3(427): 13-19.

[49] L. Chkhartishvili, D. Lezhava, O. Tsagareishvili, D. Gulua Ground-state parameters of diatomic molecules B2, BC, BN, and BO, Proc. Georgian Police Acad. 1999; 1: 195-300.

[50] L. Chkhartishvili, D. Lezhava, O. Tsagareishvili, Quasiclassical determination of electronic energies and vibration frequencies in boron compounds, J. Solid State Chem. 2000; 154(1): 148-152. https://doi.org/10.1006/jssc.2000.8826

[51] LS. Chkhartishvili, DT. Lezhava, Zero-point vibration effect on crystal binding energy: Quasi-classical calculation for laminated boron nitride, Trans. Georgian Tech. Univ. 2001; 6(439): 87-90.

[52] L. Chkhartishvili, Ground state parameters of wurtzite-like boron nitride: Quasi-classical estimations, In: Proc. 1st Int. Boron Symp. (Ed. K. Erarslan), Ankara, Dumlupinar Univ. TMMOB 2002; 139-143.

[53] L. Chkhartishvili, Quasi-classical approach: Electronic structure of cubic boron nitride crystals, J. Solid State Chem. 2004; 177(2): 395-399. https://doi.org/10.1016/j.jssc.2003.03.004

[54] L. Chkhartishvili, Quasi-classical analysis of boron-nitride binding, In: Proc. 2nd Int. Boron Symp. (Eds. H. Ozdag, H. Akdas, V. Bozkurt, M. Iphar), Ankara, Osmangazi Univ. TMMOB 2004; 165-171.

[55] LS. Chkhartishvili, Quasi-classical estimates of the lattice constant and band gap of a crystal: Two-dimensional boron nitride, Phys. Solid State 2004; 46(11): 2126-2133. https://doi.org/10.1134/1.1825560 
[56] L. Chkhartishvili, Quasi-classical analysis of electron bandwidths in wurtzite-like boron nitride. Ilia Chavchavadze Tbilisi Univ. Sci. Trans. 2005; 1: 296-314.

[57] L. Chkhartishvili, Density of electron states in wurtzite-like boron nitride: A quasi-classical calculation, Mater. Sci. Ind. J. 2006; 2(1): 18-23.

[58] L. Chkhartishvili, Zero-point vibration energy within quasiclassical approximation: Boron nitrides, Proc. Tbilisi State Univ. (Phys.) - Georgian e-Sci. J. Phys. 2006; 40: 130-138.

[59] LS. Chkhartishvili, Equilibrium geometry of ultra-small radius boron nitride nanotubes, Mater. Sci. Nanostr. 2009; 1: 33-44.

[60] L. Chkhartishvili, On quasi-classical estimations of boron nanotubes ground-state parameters, J. Phys. Conf. Ser. 2009; 176(012013): 1-9. https://doi.org/10.1088/1742-6596/176/1/012013

[61] L. Chkhartishvili, Molar binding energy of the boron nanosystems, In: Proc. 4th Int. Boron Symp. (Eds. A. Konuk, H. Kurama, H. Ak, M. Iphar), Ankara, Osmangazi Univ. TMMOB 2009; 153-160.

[62] L. Chkhartishvili, I. Murusidze, On relative stability of singlewalled boron nitride nanotubes, Nano Studies 2010; 2: 183212

https://doi.org/10.1142/9789814343909 0029

[63] L. Chkhartishvili, I. Murusidze, Molar binding energy of zigzag and armchair single-walled boron nitride nanotubes, Mater. Sci. Appl. 2010; 1(4): 223-246. https://doi.org/10.4236/msa.2010.14035

[64] L. Chkhartishvili, Geometrical models for bare boron nanotubes, In: Physics, Chemistry and Applications of Nanostructures (Eds. VE. Borisenko, SV. Gaponenko, VS. Gurin, CH. Kam), Singapore, World Scientific 2011; 118-121. https://doi.org/10.1142/9789814343909 0027

[65] L. Chkhartishvili, T. Berberashvili, I. Murusidze, Stability of small boron nitride nanotubes, In: Physics, Chemistry and Applications of Nanostructures (Eds. V.E. Borisenko, S.V. Gaponenko, V.S. Gurin, C.H. Kam), Singapore, World Scientific 2011; 126-129.

https://doi.org/10.1142/9789814343909 0029

[66] L. Chkhartishvili, Nanotubular boron: Ground-state estimates, In: New Developments in Materials Science (Eds. E. Chikoidze, T. Tchelidze), New York, Nova Sci. Publ. 2011; Ch.8: 67-80.

[67] L. Chkhartishvili, I. Murusidze, Relative stability of BN nanotubes. Solid State Sci. 2012; 14(11/12): 1664-1668. https://doi.org/10.1016/j.solidstatesciences.2012.06.005

[68] L. Chkhartishvili, I. Murusidze, M. Darchiashvili, O. Tsagareishvili, D. Gabunia, Metal impurities in crystallographic voids of beta-rhombohedral boron lattice: Binding energies and electron levels, Solid State Sci. 2012; 14(11/12): 1673-1682 https://doi.org/10.1016/j.solidstatesciences.2012.06.009

[69] L. Chkhartishvili, I. Murusidze, Thermal conductivity of $\beta$ rhombohedral boron doped with metals in nano-sized interstitials, Nano Studies 2013; 7: 221-224.

[70] L. Chkhartishvili, I. Murusidze, Frequencies of vibrations localized on interstitial metal impurities in beta-rhombohedral boron based materials, Am. J. Mater. Sci. 2014; 4(2): 103110.
[71] R. Becker, L. Chkhartishvili, R. Avci, I. Murusidze, O. Tsagareishvili, N. Maisuradze, "Metallic" boron nitride, Eur. Chem. Bull. 2015; 4(1/2/3): 8-23.

[72] L. Chkhartishvili, R. Becker, R. Avci, Relative stability of boron quasi-planar clusters, In: Proc. Int. Conf. Adv. Mater. Technol. (Eds. G. Darsavelidze, A. Guldamashvili, R. Chedia, A. Sichinava, M. Kadaria), Tbilisi, Universal 2015; 42-46.

[73] L. Chkhartishvili, Small elemental clusters in pair interaction approximation, In: Proc. 4th ICANM, Montreal, IAEMM 2016; 128-132.

[74] L. Chkhartishvili, Planar clusters of identical atoms in equilibrium: 1. Diatomic model approach. Am. J. Nano Res. Appl. 2017; 5(3-1): 1-4.

[75] L. Chkhartishvili, Boron quasi-planar clusters. A mini-review on diatomic approach. In: Proc. IEEE 7th Int. Conf. NAP, Part 4 (Ed.-in-Ch. A.D. Pogrebnjak), Sumy, Sumy State Univ. (2017) 04NESP10 1-5.

https://doi.org/10.1109/NAP.2017.8190297

[76] L. Sartinska, L. Chkhartishvili, E. Voynich, T. Eren, G. Frolov, E. Altay, I. Murusidze, O. Tsagareishvili, D. Gabunia, N Maisuradze, Effect of concentrated light on morphology and vibrational properties of boron and tantalum mixtures, Heliyon 2018; 4(3): e00585 1-14. https://doi.org/10.1016/j.heliyon.2018.e00585

[77] L. Chkhartishvili, I. Murusidze, R. Becker, Electronic structure of boron flat holeless sheet, Condensed Matter 2019; 41(28): 1-22. https://doi.org/10.3390/condmat4010028

[78] L. Chkhartishvili, Relative stability of planar clusters B11, B12, and B13 in neutral- and charged-states, Char. Appl. Nanomater. 2019; 2(761): 1-7. https://doi.org/10.24294/can.v2i2.761

[79] L. Chkhartishvili, Boron triangular sheet: Calculation of ground-state and electronic-structure parameters. In: 7th Proc. ICANM, Montreal, IAEMM 2019; 11-16.

[80] L. Chkhartishvili, D. Gabunia, O. Tsagareishvili, V. Metreveli. Estimation of isotopic composition effect on substance melting temperature, Bull. Georg. Acad. Sci. 2004; 170(3): 530-532.

[81] LS. Chkhartishvili, D.L. Gabunia, O.A. Tsagareishvili, Estimation of the isotopic effect on the melting parameters of boron, Inorg. Mater. 2007; 43(6): 594-596. https://doi.org/10.1134/S0020168507060076

[82] LS. Chkhartishvili, DL. Gabunia, OA. Tsagareishvili, Effect of the isotopic composition on the lattice parameter of boron, Powd. Metall. Met. Ceram. 2008; 47(9/10): 616-621. https://doi.org/10.1007/s11106-008-9064-9

[83] D. Gabunia, O. Tsagareishvili, L. Chkhartishvili, L. Gabunia, Isotopic composition dependences of lattice constant and thermal expansion of $\beta$-rhombohedral boron, J. Phys. Conf. Ser. 2009; 176(012022): 1-10. https://doi.org/10.1088/1742-6596/176/1/012022

[84] L. Chkhartishvili, O. Tsagareishvili, D. Gabunia, Isotopic expansion of boron, J. Metall. Eng. 2014; 3(3): 97-103. https://doi.org/10.14355/me.2014.0303.01

\section{DOI: https://doi.org/10.31875/2410-4701.2021.08.6}

(C) 2021 Levan Chkhartishvili; Zeal Press.

This is an open access article licensed under the terms of the Creative Commons Attribution Non-Commercial License (http://creativecommons.org/licenses/by-nc/3.0/) which permits unrestricted, non-commercial use, distribution and reproduction in any medium, provided the work is properly cited. 